\title{
An Eilenberg-Ganea phenomenon for actions with virtually cyclic stabilisers
}

\author{
Martin G. Fluch and Ian J. Leary
}

\begin{abstract}
In dimension 3 and above, Bredon cohomology gives an accurate purely algebraic description of the minimal dimension of the classifying space for actions of a group with stabilisers in any given family of subgroups. For some Coxeter groups and the family of virtually cyclic subgroups we show that the Bredon cohomological dimension is 2 while the Bredon geometric dimension is 3 .
\end{abstract}

Mathematics Subject Classification (2010). 57M20, 20J05, 55N25.

Keywords. Classifying spaces for families, Eilenberg-Ganea.

\section{Introduction and preliminaries}

For a discrete group $G$, a family of subgroups $\mathfrak{F}$ is a non-empty collection of subgroups of $G$ that is closed under conjugation and taking subgroups. If $\mathfrak{F}$ is a family of subgroups of $G$ then a model for $E_{\mathfrak{F}} G$, the classifying space for $G$-actions with stabilisers in $\mathfrak{F}$, is a $G$-CW-complex $X$ such that for $H \leq G$, the fixed point set $X^{H}$ is empty if $H \notin \mathfrak{F}$ and is contractible if $H \in \mathfrak{F}$. For any $G$ and $\mathfrak{F}$ there is always a model for $E_{\mathfrak{F}} G$ and it is unique up to equivariant homotopy.

In the case when $\mathfrak{F}$ consists of just the trivial group, $E_{\mathfrak{F}} G$ is the same thing as $E G$, the universal cover of an Eilenberg-Mac Lane space for $G$. In the case when $\mathfrak{F}$ is the family $\mathfrak{F}_{\text {fin }}(G)$ of all finite subgroups of $G$ (respectively the family $\mathfrak{F}_{\mathrm{vc}}(G)$ of all virtually cyclic subgroups of $G$ ) we write $\underline{E} G$ (respectively $\underline{E} G$ ) for $E_{\mathfrak{F}} G$. The minimal dimension of any model for $E_{\mathfrak{F}} G$ is denoted by $\operatorname{gd}_{\mathfrak{F}} \overline{\bar{G}}$ and is called the Bredon geometric dimension of $G$.

Homological algebra over the group ring $\mathbb{Z} G$ can be used to study models for $E G$, and Bredon cohomology is the natural generalisation for studying models for $E_{\mathfrak{F}} G$. In Bredon cohomology the orbit category $\mathcal{O}_{\mathfrak{F}} G$ replaces the group $G$. The orbit category $\mathcal{O}_{\mathfrak{F}} G$ is the category with objects the $G$-sets $G / H$ with $H \in \mathfrak{F}$ and $G$ maps as morphisms. A (right) $\mathcal{O}_{\mathfrak{F}} G$-module is then a contravariant functor from the orbit category $\mathcal{O}_{\mathfrak{F}} G$ to the category of abelian groups. In the case when $\mathfrak{F}$ consists 
of just the trivial group, $\mathcal{O}_{\mathfrak{F}} G$ is a category with one object and morphism set $G$ and $\mathcal{O}_{\mathfrak{F}} G$-modules are the same as $\mathbb{Z} G$-modules.

The category of $\mathcal{O}_{\mathfrak{F}} G$-modules is an abelian category with enough projectives. The Bredon cohomological dimension $\mathrm{cd}_{\mathfrak{F}} G$ is defined to be the projective dimension of the trivial $\mathcal{O}_{\mathfrak{F}} G$-module $\underline{\mathbb{Z}}$, which takes the value $\mathbb{Z}$ on any object of $\mathcal{O}_{\mathfrak{F}} G$ and which maps any morphism to the identity. The derived functors of the morphism functor in the category of Bredon modules over $\mathcal{O}_{\mathfrak{F}} G$ are denoted by $\operatorname{Ext}_{\mathfrak{F}}^{*}(-,-)$. The Bredon cohomology groups of $G$ with coefficients the $\mathcal{O}_{\mathfrak{F}} G$-module $M$ are the abelian groups $H_{\mathfrak{F}}^{*}(G ; M)=\operatorname{Ext}_{\mathfrak{F}}^{*}(\underline{\mathbb{Z}} ; M)$. For details on Bredon cohomology we refer to [12] or [9].

If the family $\mathfrak{F}$ consists of the trivial subgroup only, then $\operatorname{gd}_{\mathfrak{F}} G$ is the minimal dimension $\operatorname{gd} G$ an Eilenberg-Mac Lane space for $G$ can have. If $\mathfrak{F}$ is the family $\mathfrak{F}_{\text {fin }}(G)$ (respectively $\left.\mathfrak{F}_{\mathrm{vc}}(G)\right)$ then we use the notation $\underline{\operatorname{gd}} G$ (respectively $\underline{\operatorname{gd} G \text { ) }}$ for $\operatorname{gd}_{\mathfrak{F}} G$.

As in the classical case a model for $E_{\mathfrak{F}} G$ gives rise to a resolution of the trivial $\mathcal{O}_{\mathfrak{F}} G$-module $\underline{\mathbb{Z}}$ by projective $\mathcal{O}_{\mathfrak{F}} G$-modules. Therefore $\mathrm{cd}_{\mathfrak{F}} G \leq \operatorname{gd}_{\mathfrak{F}} G$ in general. If $\operatorname{cd}_{\mathfrak{F}} G \geq 3$, then $\operatorname{cd}_{\mathfrak{F}} G=\operatorname{gd}_{\mathfrak{F}} G$. In the classical case, that is when $\mathfrak{F}=\{1\}$ consists only of the trivial subgroup, this is due to Eilenberg-Ganea [7]. For $\mathfrak{F}=$ $\mathfrak{F}_{\text {fin }}(G)$ this was proved in [12] and this proof generalises to arbitrary families $\mathfrak{F}$, cf. Theorem 0.1 in [13], p. 294. In the classical case it is well known that $\operatorname{cd}_{\mathfrak{F}} G=0$ implies $\operatorname{gd}_{\mathfrak{f}} G=0$ and for general families this implication follows from Lemma 2.5 in [16], p. 265.

In the classical case, the statement that the cohomological and geometric dimension always agree is known as the Eilenberg-Ganea Conjecture. Since the work of Stallings [14] and Swan [15] implies that $\operatorname{cd} G=1$ if and only if $\operatorname{gd} G=1$, this conjecture can only be falsified by a group $G$ with cd $G=2$ but $\operatorname{gd} G=3$.

In [1] right-angled Coxeter groups $W$ such that $\underline{\mathrm{cd}} W=2$ but $\mathrm{gd} W=3$ were exhibited. We show here that some, but not all, of these examples have a similar property for actions with virtually cyclic stabilisers.

Main Theorem. Let $(W, S)$ be a right-angled Coxeter system for which the nerve $L=L(W, S)$ is an acyclic 2-complex that cannot be embedded in any contractible 2-complex.

- If $W$ is word hyperbolic, then

$$
\underline{\underline{\mathrm{cd}}} W=2 \text { and } \quad \underline{\underline{\mathrm{gd}}} W=3 .
$$

- If $W$ is not word hyperbolic, then

$$
\underline{\underline{\operatorname{cd}}} W=\underline{\underline{\operatorname{gd}}} W \geq 3 .
$$

A right angled Coxeter group $W$ is word hyperbolic if and only if its nerve $L$ satisfies the so called "flag no squares condition", cf. [4], p. 233. By Proposition 2.1 
of [5] the "flag no squares condition" puts no restriction on the homeomorphism type of the 2-complex $L$ (or see [1], p. 498, for an explicit example for a suitably triangulated $L$ ). Therefore it follows from our theorem, that the Bredon analogue of the Eilenberg-Ganea Conjecture is false for the family of virtually cyclic subgroups.

The proof of the non-word hyperbolic case of our Main Theorem is the easy part and is described in Section 3. The word hyperbolic case is Theorem 6 and 7 combined.

As mentioned before, in the classical case $\operatorname{cd}_{\mathfrak{F}} G=1$ implies $\operatorname{gd}_{\mathfrak{F}} G=1$ by the work of Stallings and Swan. It follows from Dunwoody's Accessibility Theorem [6], that the same implication is true in the case that $\mathfrak{F}=\mathfrak{F}_{\text {fin }}(G)$. In the light of this one may ask, whether this implication also holds in the case that $\mathfrak{F}=\mathfrak{F}_{\mathrm{vc}}(G)$. The first author obtained in his thesis a positive answer for countable, torsion-free, soluble groups [9], p. 127. In this class, the groups $G$ with $\underline{\underline{\mathrm{cd}}} G=1$ are precisely

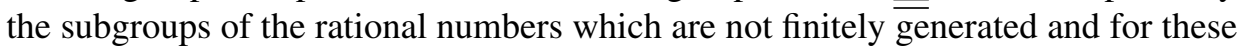
groups $\underline{\operatorname{gd}} G=1$ holds. However, a general answer to this question is still open.

Acknowledgments. The first author is grateful for the support of the CRC 701 of the DFG.

\section{Coxeter groups and the Davis complex}

A Coxeter matrix is a symmetric matrix $M=\left(m_{s t}\right)$ indexed by a finite set $S$ and with entries integers or $\infty$ subject to the conditions that for all $s, t \in S$

(1) $m_{s t}=1$ if $s=t$, and

(2) $m_{s t} \geq 2$ otherwise.

Associated to a Coxeter matrix $M$ one has the Coxeter group $W$ given by the presentation

$$
\left.W=\langle S|(s t)^{m_{s t}}=1 \text { for all } s, t \in S \text { with } m_{s t} \neq \infty\right\rangle .
$$

The Coxeter group $W$ is right-angled if the finite off-diagonal entries of the Coxeter matrix are all equal to 2 . The elements of $S$ are called the fundamental Coxeter generators of the Coxeter group $W$ and the pair $(W, S)$ is called a Coxeter system. If $T \subset S$, then $W_{T}$ denotes the subgroup of $W$ generated by $T$ and these subgroups are called special.

The nerve $L=L(W, S)$ of a Coxeter system $(W, S)$ is the simplicial complex with vertex set $S$ and whose simplices are the non-empty subsets $T \subset S$ for which the special subgroup $W_{T}$ is finite.

Given a Coxeter system $(W, S)$ the Davis complex $\Sigma=\Sigma(W, S)$ is a contractible simplicial complex on which $W$ acts with finite stabilisers; the action of the fundamental generators $S$ is by reflections. This complex has been introduced in [3] and it can interpreted as the barycentric subdivison of a cell complex where the cells are in bijective correspondence with the cosets of finite special subgroups of $W$. This cell 
complex admits in a natural way a piecewise Euclidean metric and this metric can be shown to be CAT(0). The links of the 0 -cells of this complex can be identified with the nerve $L$. The full subcomplex of $\Sigma$ whose vertices correspond to the identity cosets of the finite special subgroups is denoted by $K$. It is a fundamental domain of the action of $W$ and it can be realised as the cone of $L$, where $L$ is identified with the boundary $\partial K$ in $\Sigma$. For details see [4].

If $(W, S)$ is a right angled Coxeter system, then its nerve is a flag complex [4], p. 125. Conversely, if we are given a finite flag complex $L$, then we can construct a Coxeter system $(W, S)$ such that $L$ is its nerve as follows: let $S$ be the set of vertices of $L$ and for $s \neq t$ set $m_{s t}=2$ if $s$ and $t$ are adjacent in $L$ and set $m_{s t}=\infty$ if no edge connects $s$ and $t$ in $L$.

\section{The non-hyperbolic case}

It suffices to show that $\mathrm{cd} W \geq 3$. For this it is enough to show that $W$ contains a subgroup $H$ with $\underline{\underline{c d}} H \overline{\geq}$. Since $W$ is not word hyperbolic it contains a subgroup isomorphic to $\mathbb{Z}^{2}$ [4], p. 241.

We show that $E \mathbb{Z}^{2}=3$ using an explicit 3-dimensional model $X$ for $E \mathbb{Z}^{2}$, which was first described by Farrell. See [8] for a general construction containing this as a special case, or see [11] for a description of $X$ and a computation of $H_{*}\left(X / \mathbb{Z}^{2} ; \mathbb{Z}\right)$ from which it follows that $H^{3}\left(X / \mathbb{Z}^{2} ; \mathbb{Z}\right)$ is a countable direct product of copies of $\mathbb{Z}$. Theorem 4.2 in [9], p. 83, states that $H^{3}\left(X / \mathbb{Z}^{2}\right) \cong H_{\mathfrak{F}_{v c}\left(\mathbb{Z}^{2}\right)}^{3}\left(\mathbb{Z}^{2} ; \underline{\mathbb{Z}}\right)$. Hence it follows that $\underline{\underline{\mathrm{cd}}} \mathbb{Z}^{2}=3$.

\section{The geometric dimension in the hyperbolic case}

Given a Coxeter system $(W, S)$ and a $W$-space $X$ we set

$$
X^{\#}=\bigcup_{s \in S} X^{s}
$$

and

$$
X^{\text {sing }}=\left\{x \in X \mid W_{x} \neq 1\right\} .
$$

Clearly $X^{\#} \subset X^{\text {sing }}$.

Lemma 1. Let $K \subset \Sigma$ be the fundamental chamber of $\Sigma$ and let $s \in S$. Then both $K$ and $K \cup s K$ are convex subsets of $\Sigma$.

Proof. For each $t \in S$ the fixed point set $\Sigma^{t}$ separates $\Sigma$ into two connected half spaces. Denote by $H_{t}^{-}$the half space which does not intersect $K$ and denote by 
$\bar{H}_{t}^{+}$the complement of $H_{t}^{-}$. Then $\bar{H}_{t}^{+}$is a convex subset of $\Sigma$ containing $K$. Then $K=\bigcap_{t \in S} \bar{H}_{t}^{+}$is a convex subset of $\Sigma$. Finally, $K \cap s K$ is convex since $K \cup s K=K_{0} \cap s K_{0}$ where $K_{0}$ is the convex set $K_{0}=\bigcap_{t \in S \backslash\{s\}} \bar{H}_{t}^{+}$.

Lemma 2. Let $X$ be a model for $\underline{E} W$. Then $X^{\#}$ is homotopy equivalent to $L$.

Proof. Since $X$ is $W$-homotopy equivalent to $\Sigma$ it follows that $X^{\#}$ is homotopy equivalent to $\Sigma^{\#}$. Thus it is enough that $\Sigma^{\#}$ is homotopy equivalent to $L$.

Let $K$ be the fundamental chamber of $\Sigma$. Then $K$ is complete and compact and due to Lemma 1 also convex. Therefore, since $\Sigma$ is a CAT( 0$)$ space, there exists a retraction of $\Sigma$ onto $K$ which sends every point $x \in \Sigma \backslash K$ to the unique point $\pi(x)$ of $K$ which is nearest to $x$, cf. [2], p. 176f.

Let $K^{S}$ the union of all mirrors of $K$, that is

$$
K^{S}=\{x \in K \mid x \in K \cap s K \text { for some } s \in S\},
$$

cf. [4], p. 63, p. 127. The set $K^{S}$ is homotopy equivalent to $L$ [4], p. 127.

Let $s \in S$ and $x \in \Sigma^{\#} \backslash K$. Let $y=\pi(x)$. Then $s y \in s K$ and since $K \cup$ $s K$ is convex it follows that the midpoint $m$ of the geodesic joining $y$ and $s y$ is contained in $K \cup s K$. Since $y$ and $s y$ have the same distance from $K \cap s K$ it follows that $m \in K \cap s K$. In particular $m \in K$. Since $x \in \Sigma^{\#}$ it follows that $d(x, y)=d(s x, s y)=d(x, s y)$. Since the metric of $\Sigma$ is CAT(0) it follows that $d(x, m) \leq \max (d(x, y), d(x, s y))=d(x, y)$. By the uniqueness of the point $\pi(x)$ it follows that $m=y$. Hence $y \in K^{S}$.

It follows that the homotopy equivalence $\Sigma \simeq K$ restricts to a homotopy equivalence $\Sigma^{\#} \simeq K^{S}$. Thus $X^{\#} \simeq L$.

Remark 3. The above lemma could be used to give a slightly different proof of the main assertion of Proposition 4 of [1], p. 497.

Lemma 4. Let $X$ be a model for $\underline{\underline{E}} W$. If $W$ is word hyperbolic, then $X^{\#}$ is homotopy equivalent to

$$
L \vee \bigvee_{i \in I} S^{1}
$$

where the index set I consists of all maximal infinite virtually cyclic subgroups of $W$ which contain at least two non-commuting Coxeter generators.

Proof. Let $Y$ be the model for $\underline{E} W$ which is obtained from $\Sigma$ as described in [11]. This construction yields for every maximal infinite virtually cyclic subgroup $H$ of $W$ a 1-dimensional model $Z_{H}$ for $\underline{E} H$ together with an $H$-equivariant embedding $f_{H}: Z_{H} \rightarrow \Sigma$. We identify $Z_{H}$ with its image in $\Sigma$ under this embedding. Then $Y$ is obtained by coning off the sets $Z_{H}$ and extending the $W$-action suitably. 
Since $X$ is $W$-homotopy equivalent to $Y$ it follows that $X^{\#}$ is homotopy equivalent to $Y^{\#}$. The set $Y^{\#}$ is obtained from $\Sigma^{\#}$ by coning off the intersection $\Sigma^{\#} \cap Z_{H}$ for every maximal infinite virtually cyclic subgroup $H$ of $W$.

Let $s, t \in S$ such that $s, t \in H$ for some maximal infinite virtually cyclic subgroup $H$ of $W$. Then $x \in Z_{H}$ can be a common fixed point of $s$ and $t$ if and only if $s$ and $t$ commute. In particular $Z_{H} \cap X^{\#}$ can consist of at most 2 points as a virtually cyclic subgroup of $W$ cannot contain more than 2 pairwise non-commuting Coxeter generators. Coning off a singleton set of a path connected space does not change its homotopy type. And coning off a subset of a path connected space which has two points is homotopy equivalent to attaching a $S^{1}$ to it. Hence the claim of the lemma follows.

Lemma 5. Let $(X, A)$ be a $C W$-pair and let $B$ be a $C W$-complex which is homotopy equivalent to $A$. Then there exists a $C W$-pair $(Y, B)$ which is homotopy equivalent to $(X, A)$ such that the cells of $X \backslash A$ are dimension wise in a 1-to-1 correspondence to the cells of $Y \backslash B$.

Proof. This follows directly from Theorem 4.1.7 in [10], p. 104.

Theorem 6. Let $(W, S)$ be a Coxeter system with $W$ word hyperbolic and such that the nerve $L(W, S)$ of this Coxeter system is an acyclic complex, which is not homotopy equivalent to a subcomplex of a contractible 2-complex. Then $\mathrm{gd} W=3$.

Proof. Assume towards a contradiction that there exists a 2-dimensional model $X$ for $\underline{E} W$. Then $X^{\#}$ is homotopy equivalent to $L \vee \bigvee S^{1}$ by Lemma 4 . By Lemma 5 there exists a 2-dimensional CW-complex $Y$ which is homotopy equivalent to $X$ and which contains $L \vee \bigvee S^{1}$. In particular $L$ is a subcomplex of $Y$ contradicting the assumption that $L$ does not embed into a contractible 2-complex. Thus gd $W \geq 3$.

On the other hand, the Davis complex $\Sigma$ is a model for $\underline{E} W$ and $\operatorname{dim} \Sigma=$ $\operatorname{dim} L+1=3$. Since $W$ is word hyperbolic we can elevate $\Sigma$ to a model for $\underline{\underline{E}} W$ by attaching orbits of cells in dimension 2 and less, cf. [11]. Thus $\operatorname{gd} W \leq 3$ and equality holds.

\section{The cohomological dimension}

Theorem 7. Let $(W, S)$ be a Coxeter system with $W$ word hyperbolic and such that the nerve $L(W, S)$ of this Coxeter system is an acyclic complex which is not homotopy equivalent to a subcomplex of a contractible 2-complex. Then $\underline{\underline{\mathrm{dd}}} W=2$.

Proof. Let $\mathfrak{F}$ be the family of virtually cyclic subgroups of $W$. Let $Z$ be the submodule of the trivial $\mathcal{O}_{\mathfrak{F}} W$-module given by $Z(G / H)=\mathbb{Z}$ for any finite subgroup $H$ of $W$ and which is 0 otherwise. The complex $\Sigma^{\text {sing }}$ is acyclic and 2-dimensional 
by [1] and it follows that $\underline{C}_{*}\left(\Sigma^{\text {sing }}\right)$ gives a projective resolution of $Z$ of length 2 . Thus pd $Z \leq 2$.

On the other hand, if $X$ is a model for $\underline{E} W$, then a model $Y$ for $\underline{E} W$ can be obtained from $X$ by attaching orbits of cells in dimension 2 and less [1] , Proposition 9. It follows that $\underline{C}_{*}(Y, X)$ gives a free resolution of $Q=\underline{\mathbb{Z}} / Z$ of length 2 . Thus pd $Q \leq 2$.

Consider the short exact sequence

$$
0 \rightarrow Z \rightarrow \underline{\mathbb{Z}} \rightarrow Q \rightarrow 0
$$

of $\mathcal{O}_{\mathfrak{F}} W$-modules. Since pd $Z$ and pd $Q$ are bounded by 2 it follows by the Horseshoe Lemma that $\underline{\underline{\mathrm{pd}}} \underline{\underline{Z}} \leq 2$, that is $\underline{\underline{\mathrm{cd}}} W \leq 2$.

On the other hand, it follows from [11], Corollary 16, that the quotient space $\underline{\underline{E}} W / W$ has non-trivial cohomology in dimension 2 , and thus $H_{\mathfrak{F}}^{2}(W ; \underline{\mathbb{Z}})$ must be non-trivial too, cf. Theorem 4.2 in [9], p. 83. As a consequence we get $\underline{\underline{\mathrm{cd}}} W \geq 2$ and therefore the claim follows.

\section{References}

[1] N. Brady, I. J. Leary, and B. E. A. Nucinkis, On algebraic and geometric dimensions for groups with torsion. J. London Math. Soc. (2) 64 (2001), 489-500. Zbl 1016.20035 MR 1853466

[2] M. R. Bridson and A. Haefliger, Metric spaces of non-positive curvature. Grundlehren Math. Wiss. 319, Springer-Verlag, Berlin 1999. Zbl 0988.53001 MR 1744486

[3] M. W. Davis, Groups generated by reflections and aspherical manifolds not covered by Euclidean space. Ann. of Math. (2) 117 (1983), 293-324. Zbl 0531.57041 MR 690848

[4] M. W. Davis, The geometry and topology of Coxeter groups. London Math. Soc. Monogr. Ser. 32, Princeton University Press, Princeton, NJ, 2008. Zbl 1142.20020 MR 2360474

[5] A. N. Dranishnikov, Boundaries of Coxeter groups and simplicial complexes with given links. J. Pure Appl. Algebra 137 (1999), 139-151. Zbl 0946.20020 MR 1684267

[6] M. J. Dunwoody, Accessibility and groups of cohomological dimension one. Proc. London Math. Soc. (3) 38 (1979), 193-215. Zbl 0419.20040 MR 531159

[7] S. Eilenberg and T. Ganea, On the Lusternik-Schnirelmann category of abstract groups. Ann. of Math. (2) 65 (1957), 517-518. Zbl 0079.25401 MR 0085510

[8] F. T. Farrell and L. E. Jones, Computations of stable pseudoisotopy spaces for aspherical manifolds. In Algebraic topology Poznań 1989, Lecture Notes in Math. 1474, Springer, Berlin 1991, 59-74. Zbl 0756.57011 MR 1133892

[9] M. Fluch, On Bredon (co-)homological dimensions of groups. Ph.D. thesis, University of Southampton, 2010. arXiv:1009.4633

[10] R. Geoghegan, Topological methods in group theory. Graduate Texts in Math. 243, Springer, New York 2008. Zbl 1141.57001 MR 2365352 
[11] D. Juan-Pineda and I. J. Leary, On classifying spaces for the family of virtually cyclic subgroups. In Recent developments in algebraic topology, Contemp. Math. 407, Amer. Math. Soc., Providence, RI, 2006, 135-145. Zbl 1107.19001 MR 2248975

[12] W. Lück, Transformation groups and algebraic K-theory. Lecture Notes in Math. 1408, Springer-Verlag, Berlin 1989. Mathematica Gottingensis. Zbl 0679.57022 MR 1027600

[13] W. Lück and D. Meintrup, On the universal space for group actions with compact isotropy. In Geometry and topology: Aarhus (1998), Contemp. Math. 258, Amer. Math. Soc., Providence, RI, 2000, 293-305. Zbl 01538912 MR 1778113

[14] J. Stallings, Groups of dimension 1 are locally free. Bull. Amer. Math. Soc. 74 (1968), 361-364. Zbl 0157.05302 MR 0223439

[15] R. G. Swan, Groups of cohomological dimension one. J. Algebra 12 (1969), 585-610. Zbl 0188.07001 MR 0240177

[16] P. Symonds, The Bredon cohomology of subgroup complexes. J. Pure Appl. Algebra 199 (2005), 261-298. Zbl 1067.18013 MR 2134305

Received July 17, 2012; revised October 10, 2012

M. G. Fluch, Universität Bielefeld, Postfach 100131, 33501 Bielefeld, Germany

E-mail:mfluch@math.uni-bielefeld.de

I. J. Leary, School of Mathematics, University of Southampton, Southampton SO17 1BJ, U.K.

E-mail: I.J.Leary@ soton.ac.uk 